\title{
15-PGDH as A Therapeutic Target for Cholangiocarcinoma
}

\author{
Lu Yao*, Chang Han and Tong Wu \\ ${ }^{1}$ Department of Pathology and Laboratory Medicine, Tulane University School of Medicine, New Orleans, LA 70112, USA
}

*Corresponding author: Lu Yao, Department of Pathology and Laboratory Medicine, Tulane University School of Medicine, 1430 Tulane Avenue, SL-79, New Orleans, LA 70112, USA, Tel: 504-988-0866; E-mail: lyao1@tulane.edu

Received date: April 12, 2015, Accepted date: April 18, 2015, Published date: April 25, 2015

Copyright: $\odot 2015$ Yao L, et al. This is an open-access article distributed under the terms of the Creative Commons Attribution License, which permits unrestricted use, distribution, and reproduction in any medium, provided the original author and source are credited.

\begin{abstract}
Cholangiocarcinoma is a type of aggressive liver malignancy that arises from the biliary tract. Enhanced PGE2 signaling plays an important role in Cholangiocarcinoma development. Tumor suppressor gene 15-PGDH catalyzes oxidation of PGE2 and is potential target in intervention CCA progression. This notion is supported by our previous research that overexpression 15-PGDH inhibits CCA cells growth not only by decreasing PGE2 level, but also by activating PPARy through 15-keto-PGE2. To induce 15-PGDH expression, multiple strategies are proposed based on regulatory mechanisms of 15-PGDH. In our recent published report, we provide novel evidence that $\omega-3$ PUFA induces 15-PGDH in CCA by inhibiting miR26a/b which prevents 15-PGDH translation and support the use of $\omega$-3 PUFA as nontoxic adjuvant therapeutic agent for the treatment of human cholangiocarcinoma.
\end{abstract}

Keywords: 15-PGDH; Cholangiocarcinoma

\section{Content}

Cholangiocarcinoma (CCA) is the adenocarcinoma arising from the biliary tract, and comprises about $10 \sim 15 \%$ primary liver malignancies [1]. This type of liver cancer is extremely aggressive and has very poor prognosis. Currently, surgical resection or systemic chemotherapy is the only treatment option, although 5 -year survival associated with the treatment remains low [2]. A better understanding of CCA pathogenesis and development of specific targeted therapy is an urgent and practical need.

CCA often arises from background conditions that cause enduring inflammation, injury, and reparative biliary cell proliferation [3]. Proinflammatory and pro-proliferative prostaglandin E2 (PGE2) plays an important role in development and progression of those conditions $[1,4,5]$. Accumulated evidence has suggested PGE2 representing a promising therapeutic target for pharmacologic intervention to reduce the growth and metastatic potential of CCA [6-10].

PGE2 production is highly induced by up-regulation of cyclooxygenases-2 (COX-2) and mPGES-1 in CCA [11]. COX-2 converts arachidonic acid (AA) to prostaglandin $\mathrm{H} 2$ (PGH2) which is further transformed to PGE2 by mPGES-1. By binding to its cognate receptor (EP1-EP2), PGE2 trans-actives multiple signaling which results in enhanced cell proliferation, angiogenesis and induced invasion/motility of tumor cells [1]. We have previously reported that COX-2 derived PGE2 promotes human cholangiocarcinoma cell growth and invasion through EP1 receptor-mediated activation of the epidermal growth factor receptor and Akt. The COX-2 inhibitor celecoxib blocks phosphorylation of Akt and induces apoptosis of human cholangiocarcinoma cells [3]. In related context, overexpression of mPGES-1 in CCLP1 CCA cells increases cell proliferation, migration, invasion, and colony formation, whereas knockdown of mPGES-1 attenuates tumor growth parameters [11]. These findings demonstrate that interfering PGE2 synthesis is a promising therapeutic method in prevention and treatment of human CCA. However, a limited number of compounds have been currently addressed to inhibit PGE2 synthesis. The only FDA proved inhibitor (celecoxib) is known to be associated with increased cardiovascular side effect.

The NAD+-dependent 15-hydroxyprostaglandin dehydrogenase (15-PGDH) is the enzyme catalyzing the oxidation of the 15(S)hydroxyl group of prostaglandin E2 (PGE2), converting PGE2 to its oxidized form 15-keto-PGE2 and conferring PGE2 biological inactive [6]. However, 15-PGDH has been shown to be down-regulated in most tumors including CCA $[8,12]$. Therefore, induction of 15-PGDH expression provides another possible way to control PGE2 and treat CCA. Actually, Ectopic expression 15-PGDH in CCA cells inhibits CCA cells growth in vitro, not only because decreasing PGE2 level, but also activating PPAR $\gamma$ through 15-keto-PGE2 [8]. In our previous study, we reported that 15-ketoPGE2 act as an endogenous PPAR $\gamma$ ligand that cause SMAD 2/3 dissociation from PPAR $\gamma$ and promoted SMAD 2/3 activation. The 15-PGDH/15-keto-PGE2-induced Smad2/3 phosphorylation resulted in TAP63 auto-transcription which is mainly responsible for inhibition of CCA cells growth.

NSAIDs could inhibit PGE2 production by both inhibiting COX-2 activity and inducing 15-PGDH expression [13]. Up-regulation of 15PGDH by NSAIDs appears involving an increase in transcription and a decrease in turnover of 15-PGDH. On the other hand, it has been reported that 15-PGDH expression in tumor cell is suppressed by transcriptional repressors binding to its promoter region $[14,15]$. The repressor is mainly E-Box-Binding transcription factors including Snail, SLUG and ZEB1. In colon cancer model, it is proposed that Snail recruiting HDAC2 to 15-PGDH 5'-untranslated region to repress 15-PGDH expression [15]. To induce 15-PGDH expression, it is rationale to down-regulate these repressors expression or inhibit their activities. Yang et al. supported this notion by showing that MEK inhibitors enhanced 15-PGDH expression in lung cancer cells by down-regulation of transcriptional repressors such as SLUG and ZEB1 [14]. HDAC inhibitor could also induce 15-PGDH expression in human lung adenocarcinoma cells and colon cancer cells. Whether those drugs could be used to induce 15-PGDH expression in cholangiocarcinoma cell needs further investigation. 
In addition, 15-PGDH expression is regulated by microRNAs which prevent translation. Recently, our group reported that miR21 and miR26a controls 15-PGDH expression in cholangiocarcinoma and promotes tumor cell growth $[7,16]$. We have evidenced that those two microRNAs directly bind to 3'UTR of 15-PGDH and inhibit its translation. Up-regulated expression of those two miRNAs in cholangiocarcinoma tissue is strongly associated with decreased 15PGDH expression and severe malignancy. Knock down them enhanced 15-PGDH expression and inhibits cholangiocarcinoma cells growth in vitro and in vivo. Therefore, down-regulating those microRNAs expression is another possible way to induce 15-PGDH and treat CCA.

$\omega$-3 PUFAs, docosahexaenoic acid (DHA), and eicosapentaenoic acid (EPA) are enriched in the diets of many populations that enjoy a low incidence of cancer. These diets also obtain some modest success ameliorating advanced cancer in humans and have been widely used to inhibit carcinogenesis and tumor progression in animal models [17]. It is already known that $\omega-3$ PUFAs inhibit PGE2 signaling pathway in CCA cell lines [10]. However, the underlining mechanism is not fully illustrated. Our recent study published in Cancer Research finds $\omega-3$ PUFAs enhances the expression of 15-PGDH by down-regulation of miR26a/b [6]. Enhanced 15-PGDH expression, to a large extent, explained $\omega-3$ PUFAs induced growth inhibition of CCA cells. In the study, we discovered that treatment of human cholangiocarcinoma cells (CCLP1 and TFK-1) with $\omega-3$ PUFA (DHA) or transfection of these cells with the Fat-1 gene (encoding Caenorhabditis elegans desaturase, which converts $\omega-6$ PUFA to $\omega-3$ PUFA) significantly increased 15-PGDH expression level, but with little effect on the activity of the 15-PGDH gene promoter. Further data demonstrated that $\omega-3$ PUFA-induced down-regulation of miR26a/b expression mediated accumulation of 15-PGDH protein. Overexpression $\mathrm{miR} 26 \mathrm{a} / \mathrm{b}$ decreased 15-PGDH expression level and prevented $\omega-3$ PUFA-induced inhibition of cholangiocarcinoma cell growth. Mechanism investigation revealed that $\omega-3$ PUFA suppressed miR26a/b by inhibiting c-myc, a transcription factor that regulates miR-26/CTDSP (the family of host genes of miR26) gene clusters.

In summary, 15-PGDH is a vital tumor suppressor gene that downregulated in cholangiocarcinoma. Thus, it could be a potential therapeutic target for intervention of CCA. Mechanisms of regulation of 15-PGDH expression provoke multiple strategies to induce 15PGDH in CCA. Our results provide novel evidence that $\omega-3$ PUFA act as a non-toxic therapeutic agent to induce 15-PGDH in CCA by inhibiting miR26a/b and support the use of $\omega-3$ PUFA as nontoxic adjuvant therapeutic agent for the treatment of human cholangiocarcinoma.

\section{References}

1. $\mathrm{Wu} \mathrm{T}$ (2005) Cyclooxygenase-2 and prostaglandin signaling in cholangiocarcinoma. Biochim Biophys Acta 1755: 135-150.
2. Friman S (2011) Cholangiocarcinoma--current treatment options. Scand J Surg 100: 30-34.

3. Han C, Wu T (2005) Cyclooxygenase-2-derived prostaglandin E2 promotes human cholangiocarcinoma cell growth and invasion through EP1 receptor-mediated activation of the epidermal growth factor receptor and Akt. J Biol Chem 280: 24053-24063.

4. Nakanishi M, Rosenberg DW (2013) Multifaceted roles of PGE2 in inflammation and cancer. Semin Immunopathol 35: 123-137.

5. Wu T1 (2006) Cyclooxygenase-2 in hepatocellular carcinoma. Cancer Treat Rev 32: 28-44.

6. Yao L1, Han C1, Song K1, Zhang J1, Lim K2, et al. (2015) Omega-3 Polyunsaturated Fatty Acids Upregulate 15-PGDH Expression in Cholangiocarcinoma Cells by Inhibiting miR-26a/b Expression. Cancer Res 75: 1388-1398

7. Lu L1, Byrnes K, Han C1, Wang Y1, Wu T2 (2014) miR-21 targets 15PGDH and promotes cholangiocarcinoma growth. Mol Cancer Res 12: 890-900.

8. Lu D, Han C, Wu T (2013) 15-hydroxyprostaglandin dehydrogenasederived 15-keto-prostaglandin E2 inhibits cholangiocarcinoma cell growth through interaction with peroxisome proliferator-activated receptor-gamma, SMAD2/3, and TAP63 proteins. J Biol Chem 288: 19484-19502.

9. Lu D, Han C, Wu T (2011) Microsomal prostaglandin E synthase-1 inhibits PTEN and promotes experimental cholangiocarcinogenesis and tumor progression. Gastroenterology 140: 2084-2094.

10. Lim K, Han C, Xu L, Isse K, Demetris AJ, et al. (2008) Cyclooxygenase-2derived prostaglandin E2 activates beta-catenin in human cholangiocarcinoma cells: evidence for inhibition of these signaling pathways by omega 3 polyunsaturated fatty acids. Cancer Res 68: 553-560.

11. Jongthawin J1, Chusorn P, Techasen A, Loilome W, Boonmars T, et al. (2014) PGE2 signaling and its biosynthesis-related enzymes in cholangiocarcinoma progression. Tumour Biol 35: 8051-8064.

12. Lu D, Han C, Wu T (2014) 15-PGDH inhibits hepatocellular carcinoma growth through 15-keto-PGE2/PPAR $\hat{I}^{3}$-mediated activation of p21WAF1/Cip1. Oncogene 33: 1101-1112.

13. Tai HH, Chi X, Tong M (2011) Regulation of 15-hydroxyprostaglandin dehydrogenase (15-PGDH) by non-steroidal anti-inflammatory drugs (NSAIDs). Prostaglandins Other Lipid Mediat 96: 37-40.

14. Yang L, Amann JM, Kikuchi T, Porta R, Guix M, et al. (2007) Inhibition of epidermal growth factor receptor signaling elevates 15hydroxyprostaglandin dehydrogenase in non-small-cell lung cancer. Cancer Res 67: 5587-5593.

15. Backlund MG, Mann JR, Holla VR, Shi Q, Daikoku T, et al. (2008) Repression of 15-hydroxyprostaglandin dehydrogenase involves histone deacetylase 2 and snail in colorectal cancer. Cancer Res 68: 9331-9337.

16. Zhang J, Han C, Wu $\mathrm{T}$ (2012) MicroRNA-26a promotes cholangiocarcinoma growth by activating $\hat{\mathrm{I}}^{2}$-catenin. Gastroenterology 143: 246-256.

17. Edwards IJ, O'Flaherty JT (2008) Omega-3 Fatty Acids and PPARgamma in Cancer. PPAR Res 2008: 358052 